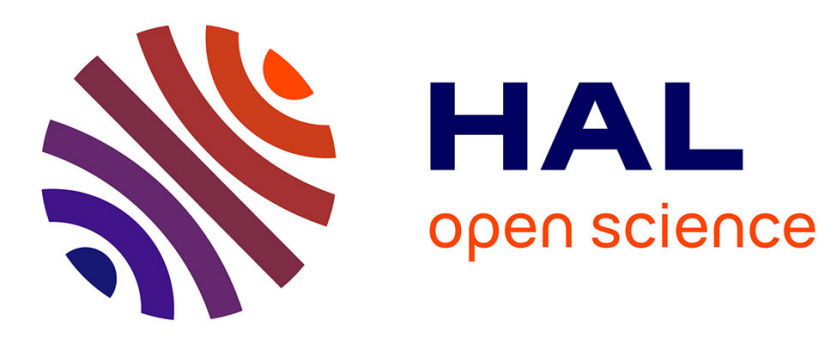

\title{
Mechanics of Viscoelastic Plates Made of FGMs
}

\author{
Holm Altenbach, Victor A. Eremeyev
}

\section{To cite this version:}

Holm Altenbach, Victor A. Eremeyev. Mechanics of Viscoelastic Plates Made of FGMs. Computational Modelling and Advanced Simulations, Springer Netherlands, pp.33-48, 2011, Computational Methods in Applied Sciences Volume 24. hal-00826449

\section{HAL Id: hal-00826449 \\ https://hal.science/hal-00826449}

Submitted on 6 Jun 2013

HAL is a multi-disciplinary open access archive for the deposit and dissemination of scientific research documents, whether they are published or not. The documents may come from teaching and research institutions in France or abroad, or from public or private research centers.
L'archive ouverte pluridisciplinaire $\mathbf{H A L}$, est destinée au dépôt et à la diffusion de documents scientifiques de niveau recherche, publiés ou non, émanant des établissements d'enseignement et de recherche français ou étrangers, des laboratoires publics ou privés. 


\title{
Mechanics of Viscoelastic Plates Made of FGMs
}

\author{
H. Altenbach and V.A. Eremeyev
}

\begin{abstract}
Considering the viscoelastic behavior of polymer foams a new plate theory based on the direct approach is introduced and applied to plates composed of functionally graded materials (FGM). The governing two-dimensional equations are formulated for a deformable surface, the viscoelastic effective stiffness parameters are identified assuming linear viscoelastic material behavior. The material properties are changing in the thickness direction. Solving some problems of the global structural analysis it will be demonstrated that in some cases the results significantly differ from the results based on the Kirchhoff-type theory. The aim of this paper is to extend the results of the analysis given in (ZAMM 88:332-341, 2008; Acta Mech 204:137-154, 2009; Key Eng Mater 399:63-70,2009) related to the case of general linear viscoelastic behaviour and to discuss how the effective viscoelastic properties reflect the properties in the thickness direction.
\end{abstract}

Keywords Plates . Viscoelasticity · Functionally graded material · Foam

\subsection{Introduction}

Foams are a very perspective class of materials for modern engineering applications. Metallic and polymeric foams are applied as a material for lightweight structures which are used in civil engineering, in the automotive or aerospace industries since they combine low weight, high specific strength, and excellent possibilities to absorb energy, see $[4,5]$. The technical realization is mostly performed as sandwich panels (plates or shells with hard and stiff face sheets and a core layer made of foam). From the mechanical point of view, foam is a very complex material, and it can be modeled with the help of three- or two-dimensional theories. Below the non-homogeneous foam is presented as a functionally graded material (FGM) 
Fig. 2.1 Non-homogeneous foam

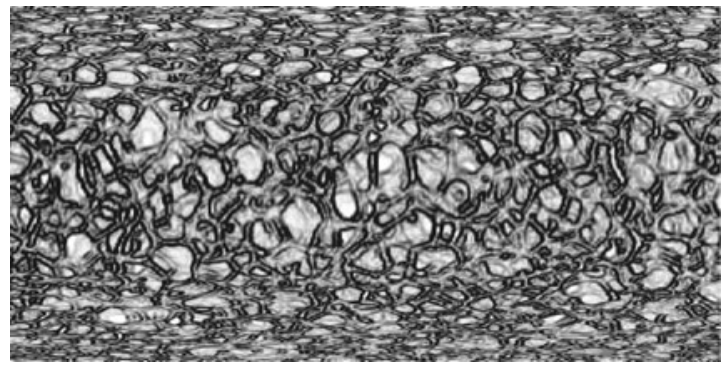

with "smeared" mechanical properties. By this way the changing over the thickness direction properties are substituted by effective stiffness parameters.

The analysis of plates and shell structures can be performed applying theories deduced by various approaches. Here we present a new theory of viscoelastic plates with changing properties in the thickness direction based on the direct approach in the plate theory and extended by the effective properties concept. We consider plates made of polymer foams with highly non-homogeneous structure through the thickness (see, for example Fig. 2.1). From the direct approach point of view a plate or a shell is modeled as a material surface each particle of which has five degrees of freedom (three displacements and two rotations, the rotation about the normal to plate is not considered as a kinematically independent variable, which corresponds to ignoring drilling moments effects). Such a model can be accepted in the case of plates with constant or slow changing thickness. For the linear variant of such theory the identification of the elastic stiffness tensors considering changing properties is proposed in [6-8], see also [9, 10]. Some extensions of the proposed theory of plates to the case of viscoelastic materials are given in [11] and applied to FGMs in [1-3].

Let us note that functionally graded plates and shells are investigated in many papers, see [12-17] among others. Different theories based on kinematical hypotheses or some mathematical treatments of the three-dimensional equations are presented. The suggested theories can be classified mostly as first order shear deformation theories or higher order theories. The first ones are based on the improvement of the strains introducing both independent translations and rotations of the points of the mid-plane of the plate while the second class of theories is very popular in the computational community. Both types of theories have advantages and disadvantages. Within the proposed theories the elastic, thermoelastic, and magnetoor electroelastic behaviour of the plate material are taken into account. In general, the viscoelastic properties of FGM plates are less considered see [1-3] and [18].

Here we focus our attention on the viscoelastic behavior. The dependence of the relaxation functions on the plate through-the-thickness structure as well as on the bulk material viscoelastic properties is analyzed. We show that for FGM plates the effective viscoelastic properties significantly depend on the bulk properties as functions of the thickness coordinate $z$. In particular, we discuss the influence of the plate geometry on the spectrum of the relaxation time. As a special case the viscoelastic behavior of a sandwich plate with a core made of a FGM is considered. 


\subsection{Governing Equations of a 5-Parametric Plate Theory}

Let us consider the geometrically and physically linear theory of plates. The equations of motion are formulated as the Euler's laws of dynamics

$$
\begin{gathered}
\nabla \cdot \mathbf{T}+\mathbf{q}=\rho \ddot{\mathbf{u}}+\rho \boldsymbol{\Theta}_{1} \cdot \ddot{\boldsymbol{\varphi}}, \\
\nabla \cdot \mathbf{M}+\mathbf{T}_{\times}+\mathbf{m}=\rho \boldsymbol{\Theta}_{1}^{T} \cdot \ddot{\mathbf{u}}+\rho \boldsymbol{\Theta}_{2} \cdot \ddot{\varphi} .
\end{gathered}
$$

Here $\mathbf{u}$ and $\varphi$ are the vectors of displacements and rotations,

$$
\mathbf{u}=u_{1} \mathbf{e}_{1}+u_{2} \mathbf{e}_{2}+w \mathbf{n}, \varphi=-\varphi_{2} \mathbf{e}_{1}+\varphi_{1} \mathbf{e}_{2},
$$

$\mathbf{n}$ is the unit outer normal vector at the plate surface, $\mathbf{e}_{1}, \mathbf{e}_{2}$ are orthonormal vectors in the tangent plane, $\mathbf{T}$ and $\mathbf{M}$ are the tensors of forces and moments,

$$
\begin{gathered}
\mathbf{T}=T_{1} \mathbf{e}_{1} \mathbf{e}_{1}+T_{2} \mathbf{e}_{2} \mathbf{e}_{2}+T_{12}\left(\mathbf{e}_{1} \mathbf{e}_{2}+\mathbf{e}_{2} \mathbf{e}_{1}\right)+T_{1 n} \mathbf{e}_{1} \mathbf{n}+T_{2 n} \mathbf{e}_{2} \mathbf{n}, \\
\mathbf{M}=M_{1} \mathbf{e}_{1} \mathbf{e}_{2}-M_{2} \mathbf{e}_{2} \mathbf{e}_{1}-M_{12}\left(\mathbf{e}_{1} \mathbf{e}_{1}-\mathbf{e}_{2} \mathbf{e}_{2}\right),
\end{gathered}
$$

$\mathbf{q}$ and $\mathbf{m}$ are the vectors of surface loads (forces and moments), $\mathbf{T}_{\times}$is the vector invariant of the force tensor, $\nabla$ is the nabla (Hamilton) operator, $\boldsymbol{\Theta}_{\mathbf{1}}$ and $\boldsymbol{\Theta}_{\mathbf{2}}$ are the first and the second tensor of inertia, $\rho$ is the density (effective property of the deformable surface), the upper index $\mathrm{T}$ denotes transposed, and $(\cdots)^{\circ}$ the time derivative, respectively.

In the case of orthotropic behavior the following constitutive equations for the stress resultants are valid:

- In-plane forces

$$
\mathbf{T} \cdot \mathbf{a}=\int_{-\infty}^{t} \mathbf{A}(t-\tau) \cdot \dot{\boldsymbol{\mu}} d \tau+\int_{-\infty}^{t} \mathbf{B}(t-\tau) \cdot \dot{\mathbf{K}} d \tau,
$$

- Transverse shear forces

$$
\mathbf{T} \cdot \mathbf{n}=\int_{-\infty}^{t} \boldsymbol{\Gamma}(t-\tau) \cdot \dot{\gamma} d \tau
$$

- Moments

$$
\mathbf{M}^{T}=\int_{-\infty}^{t} \dot{\mu} \cdot \mathbf{B}(t-\tau) d \tau+\int_{-\infty}^{t} \mathbf{C}(t-\tau) \cdot \dot{\kappa} d \tau,
$$

where $\mathbf{A}, \mathbf{B}, \mathbf{C}$ are fourth-order tensors, and $\boldsymbol{\Gamma}$ is a second-order tensor. They describe the relaxation functions of the plate, $\mu, \kappa$, and $\gamma$ are the tensor of in-plane 
strains, the tensor of the out-of-plane strains, and the vector of transverse shear strains, respectively. They are given by the relations

$$
2 \mu=\nabla \mathbf{u}+(\nabla \mathbf{u})^{T}, \kappa=\nabla \varphi, \gamma=\nabla \mathbf{u}-\mathbf{a} \times \varphi .
$$

Here $\mathbf{a}$ is the first metric tensor. The component form of (2.9) is given by

$$
\begin{gathered}
\boldsymbol{\mu}=\mu_{11} \mathbf{e}_{1} \mathbf{e}_{1}+\mu_{22} \mathbf{e}_{2} \mathbf{e}_{2}+\mu_{12}\left(\mathbf{e}_{1} \mathbf{e}_{2}+\mathbf{e}_{2} \mathbf{e}_{1}\right), \\
\kappa=\kappa_{11} \mathbf{e}_{1} \mathbf{e}_{2}-\kappa_{12} \mathbf{e}_{1} \mathbf{e}_{1}+\kappa_{21} \mathbf{e}_{2} \mathbf{e}_{2}-\kappa_{22} \mathbf{e}_{2} \mathbf{e}_{1}, \\
\boldsymbol{\gamma}=\gamma_{1} \mathbf{e}_{1}+\gamma_{2} \mathbf{e}_{2} .
\end{gathered}
$$

In Cartesian coordinates the constitutive equations are

$$
\begin{gathered}
T_{\alpha \beta}=\int_{-\infty}^{t} A_{\alpha \beta \gamma \delta}(t-\tau) \dot{\mu}_{\gamma \delta}(\tau) d \tau+\int_{-\infty}^{t} B_{\alpha \beta \gamma \delta}(t-\tau) \dot{\kappa}_{\gamma \delta}(\tau) d \tau, \\
T_{\alpha 3}=\int_{-\infty}^{t} \Gamma_{\alpha \beta}(t-\tau) \dot{\gamma}_{\beta}(\tau) d \tau, \\
M_{\alpha \beta}=\int_{-\infty}^{t} B_{\alpha \beta \gamma \delta}(t-\tau) \dot{\mu}_{\gamma \delta}(\tau) d \tau+\int_{-\infty}^{t} C_{\alpha \beta \gamma \delta}(t-\tau) \dot{\kappa}_{\gamma \delta}(\tau) d \tau,
\end{gathered}
$$

with

$$
\begin{aligned}
& \mu_{11}=u_{1,1}, \mu_{22}=u_{2,2}, 2 \mu_{12}=u_{1,2}+u_{2,1}, \\
& \kappa_{11}=\varphi_{1,1}, \kappa_{22}=\varphi_{2,2}, \kappa_{12}=\varphi_{2,1}, \kappa_{21}=\varphi_{1,2}, \\
& \gamma_{1}=w_{, 1+} \varphi_{1}, \gamma_{2}=w_{, 2+} \varphi_{2},
\end{aligned}
$$

where $T_{\alpha \beta}$ are the in-plane forces, $T_{\alpha 3}$ are the transverse shear forces, $M_{\alpha \beta}$ are the moments, $A_{\alpha \beta \gamma \delta}, B_{\alpha \beta \gamma \delta}, C_{\alpha \beta \gamma \delta}$ and $\Gamma_{\alpha \beta}$ are the relaxation functions for the plate, $\alpha, \beta, \gamma, \delta=1,2, \mu_{11}, \mu_{22}$ are the in-plane normal strains, $\mu_{12}$ is the in-plane shear strain, $\gamma_{\alpha}$ are the transverse shear strains, $\kappa_{11,} \kappa_{22}$ are the bending deformations and $\kappa_{12}$ is the twist deformation.

For the orthotropic material behavior the effective relaxation tensors have the form

$$
\begin{aligned}
& \mathbf{A}=A_{11} \mathbf{a}_{1} \mathbf{a}_{1}+A_{12}\left(\mathbf{a}_{1} \mathbf{a}_{2}+\mathbf{a}_{2} \mathbf{a}_{1}\right)+A_{22} \mathbf{a}_{2} \mathbf{a}_{2}+A_{44} \mathbf{a}_{4} \mathbf{a}_{4}, \\
& \mathbf{B}=B_{13} \mathbf{a}_{1} \mathbf{a}_{3}+B_{14} \mathbf{a}_{1} \mathbf{a}_{4}+B_{23} \mathbf{a}_{2} \mathbf{a}_{3}+B_{24} \mathbf{a}_{2} \mathbf{a}_{4}+B_{42} \mathbf{a}_{4} \mathbf{a}_{2}, \\
& \mathbf{C}=C_{22} \mathbf{a}_{2} \mathbf{a}_{2}+C_{33} \mathbf{a}_{3} \mathbf{a}_{3}+C_{34}\left(\mathbf{a}_{3} \mathbf{a}_{4}+\mathbf{a}_{4} \mathbf{a}_{3}\right)+C_{44} \mathbf{a}_{4} \mathbf{a}_{4}, \\
& \Gamma=\Gamma_{1} \mathbf{a}_{1}+\Gamma_{2} \mathbf{a}_{2}
\end{aligned}
$$

with $\left(\mathbf{a}_{1} ; \mathbf{a}_{2}\right)=\mathbf{e}_{1} \mathbf{e}_{1} \pm \mathbf{e}_{2} \mathbf{e}_{2},\left(\mathbf{a}_{3} ; \mathbf{a}_{4}\right)=\mathbf{e}_{1} \mathbf{e}_{2} \mp \mathbf{e}_{2} \mathbf{e}_{1}$. 
In the case of isotropic and symmetric over the thickness plates the effective relaxation tensors have a reduced structure

$$
\begin{gathered}
\mathbf{A}=A_{11} \mathbf{a}_{1} \mathbf{a}_{1}+A_{22}\left(\mathbf{a}_{2} \mathbf{a}_{2}+\mathbf{a}_{4} \mathbf{a}_{4}\right), \mathbf{B}=\mathbf{0}, \\
\mathbf{C}=C_{22} \mathbf{a}_{2} \mathbf{a}_{2}+C_{44}\left(\mathbf{a}_{3} \mathbf{a}_{3}+\mathbf{a}_{4} \mathbf{a}_{4}\right), \Gamma=\Gamma \mathbf{a} .
\end{gathered}
$$

\subsection{Effective Properties}

Using the same technique as for the elastic plates (see, for example [10]) below we compute the viscoelastic stiffness tensor components. Let us consider the threedimensional viscoelastic constitutive equations

$$
\boldsymbol{\sigma}=\int_{-\infty}^{t} \mathbf{R}(t-\tau) \cdot \dot{\varepsilon} d \tau,
$$

or in the inverse form

$$
\boldsymbol{\varepsilon}=\int_{-\infty}^{t} \mathbf{J}(t-\tau) \cdot \dot{\boldsymbol{\sigma}} d \tau,
$$

where $\sigma$ and $\varepsilon$ are the stress and strain tensors, $\mathbf{R}$ and $\mathbf{J}$ are the 4th order tensors of relaxation and creep functions, respectively.

Further we consider two cases:

- Case 1. Homogeneous plates - all properties are constant (no dependency of the thickness coordinate $z$ ).

- Case 2. Inhomogeneous plates (sandwich, multilayered, functionally graded) all properties are functions of $z$ only.

These means that in the general case $\mathbf{R}$ and $\mathbf{J}$ depend on the thickness coordinate $z$ and on the time $t$.

Using the Laplace transform

$$
\bar{f}(s)=\int_{-\infty}^{t} f(t) e^{-s t} d t,
$$

of a function $f(t)$ one can write (2.10) and (2.11) as follows

$$
\overline{\boldsymbol{\sigma}}=s \overline{\mathbf{R}} \cdot \bar{\varepsilon}, \bar{\varepsilon}=s \overline{\mathbf{J}} \cdot \overline{\boldsymbol{\sigma}} .
$$

Using the correspondence principle (the analogy between (2.12) and the Hooke's law) we can extend the identification procedure presented in [6-8] to the Laplace 
mapping of the effective relaxation or creep functions, see [1,3]. For the orthotropic viscoelastic material the in-plane and the out-of-plane stiffness tensor components are

$$
\begin{array}{ll}
\left(\bar{A}_{11} ; \bar{B}_{13} ; \bar{C}_{33}\right) & =\frac{1}{4}\left\langle\frac{\bar{E}_{1}+\bar{E}_{2}+2 \bar{E}_{1} \bar{v}_{12}}{1-\bar{v}_{12} \bar{v}_{21}}\left(1 ; z ; z^{2}\right)\right\rangle, \\
\left(\bar{A}_{12} ;-\bar{B}_{23}=\bar{B}_{14} ; \bar{C}_{34}\right) & =\frac{1}{4}\left\langle\frac{\bar{E}_{1}-\bar{E}_{2}}{1-\bar{v}_{12} \bar{v}_{21}}\left(1 ; z ; z^{2}\right)\right\rangle, \\
\left(\bar{A}_{22} ;-\bar{B}_{24} ; \bar{C}_{44}\right) & =\frac{1}{4}\left\langle\frac{\bar{E}_{1}+\bar{E}_{2}-2 \bar{E}_{1} \bar{v}_{21}}{1-\bar{v}_{12} \bar{v}_{21}}\left(1 ; z ; z^{2}\right)\right\rangle, \\
\left(\bar{A}_{44} ; \bar{B}_{42} ; \bar{C}_{22}\right) & =\frac{1}{4}\left\langle\bar{G}_{12}\left(1 ; z ; z^{2}\right)\right\rangle,
\end{array}
$$

while the transverse shear relaxation tensor components are

$$
\begin{aligned}
& \bar{\Gamma}_{1}=\frac{1}{2}\left(\lambda^{2}+\eta^{2}\right) \frac{\bar{A}_{44} \bar{C}_{22}-\bar{B}_{42}^{2}}{\bar{A}_{44}}, \\
& \bar{\Gamma}_{2}=\frac{1}{2}\left(\lambda^{2}-\eta^{2}\right) \frac{\bar{A}_{44} \bar{C}_{22}-\bar{B}_{42}^{2}}{\bar{A}_{44}},
\end{aligned}
$$

where $\lambda$ and $\eta$ are the minimal nonzero eigen-values following from the SturmLiouville problems

$$
\begin{array}{ll}
\frac{d}{d z}\left(\bar{G}_{2 n} \frac{d Z}{d z}\right)+\lambda^{2} \bar{G}_{12} Z=0, & \left.\frac{d Z}{d z}\right|_{|z|=h / 2}=0, \\
\frac{d}{d z}\left(\bar{G}_{1 n} \frac{d \tilde{Z}}{d z}\right)+\lambda^{2} \bar{G}_{12} \tilde{Z}=0, & \left.\frac{d \tilde{Z}}{d z}\right|_{|z|=h / 2}=0 .
\end{array}
$$

Here $h$ is the plate thickness and $\langle(\cdots)\rangle$ denotes integration over the thickness.

In the case of isotropic material behaviour these formulas are simplified, see [1] for details. The non-zero components of the relaxation tensors are given by

- the in-plane relaxation functions

$$
\bar{A}_{11}=\frac{1}{2}\left\langle\frac{\bar{E}}{1-\bar{v}}\right\rangle, \bar{A}_{22}=\frac{1}{2}\left\langle\frac{\bar{E}}{1+\bar{v}}\right\rangle=\bar{A}_{44}=\langle\bar{G}\rangle,
$$

- the coupling relaxation functions

$$
\begin{aligned}
& \bar{B}_{13}=-\frac{1}{2}\left\langle\frac{\bar{E}_{1}+\bar{E}_{2}+2 \bar{E}_{1} \bar{\nu}_{21}}{1-\bar{v}_{12} \bar{v}_{21}} z\right\rangle, \\
& \bar{B}_{24}=\frac{1}{2}\left\langle\frac{\bar{E}}{1+\bar{v}} z\right\rangle=-\bar{B}_{42}=\langle\bar{G} z\rangle,
\end{aligned}
$$


- the out-of-plane relaxation functions

$$
\begin{aligned}
& \bar{C}_{33}=\frac{1}{2}\left\langle\frac{\bar{E}}{1-\bar{v}} z^{2}\right\rangle, \\
& \bar{C}_{22}=\frac{1}{2}\left\langle\frac{\bar{E}}{1+\bar{v}} z^{2}\right\rangle=\bar{C}_{44}=\left\langle\bar{G} z^{2}\right\rangle,
\end{aligned}
$$

- the transverse shear relaxation function

$$
\bar{\Gamma}_{1}=\bar{\Gamma}=\lambda^{2} \frac{\bar{A}_{44} \bar{C}_{22}-\bar{B}_{42}^{2}}{\bar{A}_{44}},
$$

with $\lambda$ following from

$$
\frac{d}{d z}\left(\bar{G} \frac{d Z}{d z}\right)+\lambda^{2} \bar{G} Z=0,\left.\quad \frac{d Z}{d z}\right|_{|z|=h / 2}=0 .
$$

For the plate which is symmetrically to the mid-plane the relation $\mathbf{B}=\mathbf{0}$ holds true. The relaxation functions of the isotropic viscoelastic plate with symmetric cross-section were considered in [1]. Note that for isotropic viscoelastic material we introduced three functions $\bar{E}(s), \bar{G}(s)$ and $\bar{v}(s)$. They are interlinked by the formula

$$
\bar{E}(s)=2 \bar{G}(s)(1+\bar{v}(s)) .
$$

Following $[19,20]$ we use (2.14) as the definition of the Poisson's ratio for isotropic viscoelastic material.

In the theory of viscoelasticity of solids the assumption $v(t)=v=$ const is often used. It is fulfilled in many applications (see arguments in [21-23] concerning $v(t) \approx$ const), for example, $v=0.5$ for an incompressible viscoelastic material. In the general case, $v$ is a function of $t . v(t)$ is assumed to be an increasing function of $t$ [23-25] or non-monotonous function of $t$, see [19, 20]. The latter case may be realized for cellular materials or foams. Further we consider the influence of $v(t)$ on the deflection of viscoelastic plate and its effective relaxation functions.

\subsection{Example of Effective Properties}

\subsubsection{Homogeneous Plate}

The simplest test for the correctness of the estimated stiffness properties is the homogeneous isotropic plate. The basic geometrical property is the thickness $h$. The plate is symmetrically with respect to the mid-plane. All material properties are constant over the thickness, i.e. they do not depend on the thickness coordinate $z$. 
For the sake of simplicity, at first let us first consider the case $v(t)=v=$ const. This means that the following relation is held true: $E(t)=2 G(t)(1+v)$. The non-zero components of the classical tensors are

$$
\begin{gathered}
A_{11}(t)=\frac{E(t) h}{2(1-v)}, A_{22}(t)=\frac{E(t) h}{2(1+v)}=G(t) h, \\
C_{33}(t)=\frac{E(t) h^{3}}{24(1-v)}, C_{22}(t)=\frac{E(t) h^{3}}{24(1+v)}=\frac{G(t) h^{3}}{12} .
\end{gathered}
$$

The bending stiffness $D$ results in

$$
D(t)=\frac{E(t) h^{3}}{12\left(1-v^{2}\right)} .
$$

The transverse shear relaxation function follows from (2.13). The solution of (2.13) yields the smallest eigen-value $\lambda=\pi / h$ which does not depend on $s$. Finally, one obtains

$$
\Gamma(t)=\frac{\pi^{2}}{12} G(t) h
$$

$\pi^{2} / 12$ is a factor similar to the shear correction factor which was first introduced by Timoshenko in the theory of beams [26]. Here this factor is a result of the nonclassical establishments of the transverse shear stiffness. Comparing this value with the Mindlin's estimate $\pi^{2} / 12$ and the Reissner's estimate 5/6 one concludes that the direct approach yields in the same value like in Mindlin's theory (note that Mindlin's shear correction is based on the solution of a dynamic problem, here was used the solution of a quasi-static problem), see [27-30]. The Reissner's value slightly differs. The graphs of $D(t)$ are given in Fig. 2.2 for two values of $v$, i.e. for $v=0.1 ; 0.4$. Let us note that in this case $D(t)$ and $\Gamma(t)$ demonstrate the same spectrum of relaxation times as the bulk material.

At second, let us consider the general case $v=v(t)$. Using the convolution theorem [21-25] in this case $D$ is reconstructed from

$$
\bar{D}(s)=\frac{\bar{E}(s) h^{3}}{12\left[1-\bar{v}^{2}(s)\right]}
$$

as follows

$$
D(t)=\int_{-\infty}^{t} \frac{E(t-\tau) h^{3}}{12\left[1-v^{2}(\tau)\right]} d \tau .
$$

Using the initial and the final value theorems [21-25]

$$
f(0)=\lim _{s \rightarrow \infty} \bar{f}(s), \quad f(\infty) \equiv \lim _{t \rightarrow \infty} f(t)=\lim _{s \rightarrow 0} \bar{f}(s)
$$




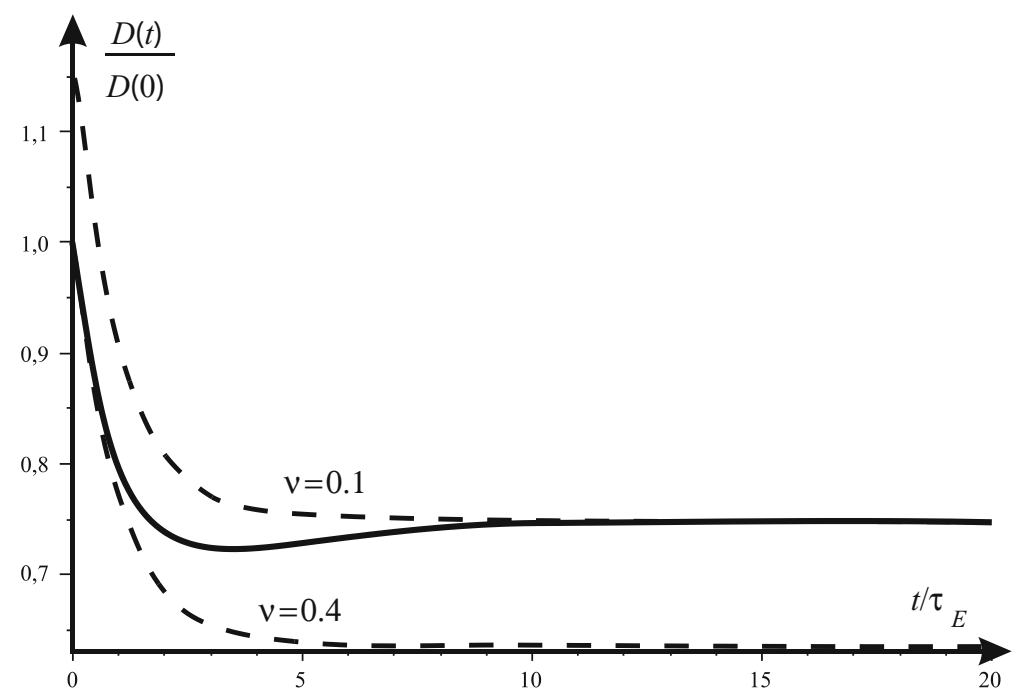

Fig. 2.2 Dimensionless bending stiffness in dependence on time: General case - solid line; Constant Poisson's ratio - dashed lines

we establish that

$$
D(0)=\frac{E(0) h^{3}}{12\left[1-v^{2}(0)\right]}, D(\infty)=\frac{E(\infty) h^{3}}{12\left[1-v^{2}(\infty)\right]} .
$$

where the values

$$
\nu(0)=\frac{E(0)}{2 G(0)}-1, \nu(\infty)=\frac{E(\infty)}{2 G(\infty)}-1
$$

may be considered as the Poisson's ratio in the initial and the relaxed state, respectively. In the general case $v=v(t)$ the relaxation function $D(t)$ is a non-monotonous function of $t$, while $D(t)$ is a monotonous decreasing function for constant Poisson's ratio, see Fig. 2.2. Here $v(0)=0.4, v(\infty)=0.1$. This means that in general case $D(t)$ and $\Gamma(t)$ demonstrate the spectrum of relaxation times which is not coincident with the spectrum of bulk material.

\subsubsection{FGM Plate}

In this section we consider small deformations of a FGM plate made of a viscoelastic polymer foam. For the panel made of a porous polymer foam the distribution of the pores over the thickness can be inhomogeneous (see, for example, Fig. 2.1). Let us introduce $\rho_{s}$ as the density of the bulk material and $\rho_{p}$ as the minimum value of the density of the foam. For the description of the symmetric distribution of the porosity 
we assume the power law [2]

$$
V(z)=\alpha+(1-\alpha)\left|\frac{2 z}{h}\right|^{n},
$$

where $\alpha=\rho_{p} / \rho_{s}$ is the minimal relative density. $n=0$ corresponds to the homogeneous plate described in the previous paragraph.

The properties of the foam strongly depend on the porosity and the cell structure. For the polymer foam in $[4,5]$ the modification of the standard linear viscoelastic solid is proposed. For the open-cell foam the constitutive law has the form

$$
\dot{\sigma}+\tau_{E} \sigma=C_{1} V^{2}(z)\left[E_{\infty} \tau_{E} \varepsilon+E_{0} \dot{\varepsilon}\right]
$$

while for the closed-cell foam the constitutive equation has the form

$$
\dot{\sigma}+\tau_{E} \sigma=C_{2}\left[\phi^{2} V^{2}(z)+(1-\phi) V(z)\right]\left[E_{\infty} \tau_{E} \varepsilon+E_{0} \dot{\varepsilon}\right]
$$

Here $C_{1} \approx 1, C_{2} \approx 1, \phi$ describes the relative volume of the solid polymer concentrated near the cell ribs. Usually, $\phi=0.6 \ldots 0.7 . E_{\infty}, E_{0}, \tau_{E}$ are material constants of the polymer used in manufacturing of the foam.

From (2.17) and (2.18) one can see that the corresponding relaxation function is given by the relations

$$
E=E(z, t)=E(t) k(z),
$$

where $E(t)$ is defined by

$$
E(t)=E_{\infty}+\left(E_{0}-E_{\infty}\right) e^{-t / \tau_{E}},
$$

while

$$
k(z)=C_{1} V^{2}(z)
$$

for open-cell foam and

$$
k(z)=C_{2}\left[\phi^{2} V^{2}(z)+(1-\phi) V(z)\right]
$$

for closed-cell foam, respectively. Analogous to (2.19) the following relation can be established for the shear relaxation function

$$
G=G(z, t)=G(t) m(z)
$$

Equations (2.19) and (2.20) state that the viscoelastic properties of the foam, for example, the time of relaxation do not depend on the porosity distribution. Note that representations (2.19) and (2.20) are only simple assumptions for spatial nonhomogeneous foams.

Using experimental data presented in [5] one can assume $v(t)=v=$ const. In this case we have the relations [3] 


$$
A_{11}=A_{22} \frac{1+v}{1-v}, C_{33}=C_{22} \frac{1+v}{1-v}
$$

$A_{22}$ and $C_{22}$ are given by the relations

$$
\begin{aligned}
& A_{22}=h\left[\alpha^{2}+\frac{2 \alpha(1-\alpha)}{n+1}+\frac{(1-\alpha)^{2}}{2 n+1}\right] G(t), \\
& C_{22}=\frac{h^{3}}{12}\left[\alpha^{2}+\frac{6 \alpha(1-\alpha)}{n+3}+\frac{3(1-\alpha)^{2}}{2 n+3}\right] G(t),
\end{aligned}
$$

for the open-cell foam, and

$$
\begin{aligned}
A_{22}= & h\left\{\phi^{2}\left[\alpha^{2}+\frac{2 \alpha(1-\alpha)}{n+1}+\frac{(1-\alpha)^{2}}{2 n+1}\right]\right. \\
& \left.+(1-\phi)\left[\alpha+\frac{1-\alpha}{n+1}\right]\right\} G(t), \\
C_{22}= & \frac{h^{3}}{12}\left\{\phi^{2}\left[\alpha^{2}+\frac{6 \alpha(1-\alpha)}{n+3}+\frac{3(1-\alpha)^{2}}{2 n+3}\right]\right. \\
& \left.+(1-\phi)\left[\alpha+\frac{3(1-\alpha)}{n+3}\right]\right\} G(t)
\end{aligned}
$$

for the closed-cell foam, respectively. Here we assume that $C_{1}=C_{2}=1$, and that $\phi$ does not depend on $z$.

From (2.21), (2.22), (2.23), (2.24) and (2.25) it is easy to see that the classical relaxation functions differ only by factors from the shear relaxation function. Note that one can easily extend (2.17) and (2.18) to the case of general constitutive equations, used in the linear viscoelasticity [13-15]. Thus taking into account the assumption that $v=$ const, one can calculate the classical effective stiffness relaxation functions for general viscoelastic constitutive equations multiplying the shear relaxation function $G(t)$ with the corresponding factor similar to (2.22), (2.23), (2.24) and (2.25). In the general situation and taking into account other viscoelastic phenomena, for example, the filtration of a fluid in the saturated foam, the effective stiffness relaxation functions may be more complex than for the pure solid polymer discussed here.

Finally, we should mention that in the case of constant Poisson's ratio and with the assumption (2.19) and (2.20) the determination of the effective in-plane, bending and transverse shear stiffness tensors of a symmetric FGM viscoelastic plate made of a polymer foam can be realized by the same method as for elastic plates [9-12]. The relaxation functions for viscoelastic FGM plates can be found from the values of the corresponding effective stiffness of an elastic FGM plate by multiplication with the normalized shear relaxation function of the polymer solid. 


\subsubsection{Sandwich Plate}

Sandwich structures with a core made of foam have various applications in the engineering. Let us consider a sandwich plate with the following geometry: $h_{c}$ is the core thickness and $h_{f}$ the thickness of the face sheets $\left(h_{f}<<h_{c}\right)$. The material properties of the core and the face sheets are given by the relaxation functions $E_{c}(t), E_{f}(t)$, $G_{c}(t)$ and $G_{f}(t)$ with $E_{c}(t)<<E_{f}(t)$ and $G_{c}(t)<<G_{f}(t)$. We have again a symmetry with respect to the mid-plane that means $\mathbf{B}=\mathbf{0}$. With the thickness $h=h_{c}+h_{f}$ one gets

$$
\begin{aligned}
& \bar{A}_{11}=\frac{1}{2}\left(\frac{\bar{E}_{f} h_{f}}{1-\bar{v}_{f}}+\frac{\bar{E}_{c} h_{c}}{1-\bar{v}_{c}}\right), \\
& \bar{A}_{11}=\frac{1}{2}\left(\frac{\bar{E}_{f} h_{f}}{1+\bar{v}_{f}}+\frac{\bar{E}_{c} h_{c}}{1+\bar{v}_{c}}\right)=\bar{A}_{44}, \\
& \bar{C}_{33}=\frac{1}{24}\left[\frac{\bar{E}_{f}\left(h^{3}-h_{c}^{3}\right)}{1-\bar{v}_{f}}+\frac{\bar{E}_{c} h_{c}^{3}}{1-\bar{v}_{c}}\right], \\
& \bar{C}_{44}=\frac{1}{24}\left[\frac{\bar{E}_{f}\left(h^{3}-h_{c}^{3}\right)}{1+\bar{v}_{f}}+\frac{\bar{E}_{c} h_{c}^{3}}{1+\bar{v}_{c}}\right]=\bar{C}_{22},
\end{aligned}
$$

The bending relaxation function results in

$$
\bar{D}=\frac{1}{12}\left[\frac{\bar{E}_{f}\left(h^{3}-h_{c}^{3}\right)}{1-\bar{v}_{f}^{2}}+\frac{\bar{E}_{c} h_{c}^{3}}{1-\bar{v}_{c}^{2}}\right] .
$$

Let us consider the latter relation in detail. For the sake of simplicity we assume that the Poisson ratios are constant. Then we obtain the relation

$$
D(t)=\frac{1}{12}\left[\frac{E_{f}(t)\left(h^{3}-h_{c}^{3}\right)}{1-v_{f}^{2}}+\frac{E_{c}(t) h_{c}^{3}}{1-v_{c}^{2}}\right] .
$$

Considering the simplest form for the bulk relaxation functions, i.e. the standard visoelastic body model with

$$
\begin{aligned}
& E_{f}(t)=E_{\infty}^{f}+\left(E_{0}^{f}-E_{\infty}^{f}\right) e^{-t / \tau_{E}^{f},} \\
& E_{c}(t)=E_{\infty}^{c}+\left(E_{0}^{c}-E_{\infty}^{c}\right) e^{-t / \tau_{E}^{c}},
\end{aligned}
$$

then we immediately obtain that $D$ has two times of relaxation $\tau_{E}^{f}$ and $\tau_{E}^{c}$.

A typical sandwich structure has a very weak core. In this case the bending relaxation function and the transverse shear relaxation function can be approximated by

$$
\bar{D}=\frac{1}{4} \frac{\bar{E}_{f} h^{2} h_{f}}{1-\bar{v}_{f}^{2}}, \bar{\Gamma}=\bar{G}_{c} h,
$$


see the details given in [7-9] for the elastic case. Let us note that for such approximation the bending relaxation function $D$ is determined by the viscoelastic properties of the faces while the transverse shear relaxation function $\Gamma$ depends on the viscoelastic behaviour of the core only. For example, if the faces are made of an elastic material and the core shows the viscoelastic behavior then $D$ is constant while $\Gamma$ is a function of time

$$
D=\frac{1}{4} \frac{E_{f} h^{2} h_{f}}{1-v_{f}^{2}}, \Gamma(t)=G_{c}(t) h .
$$

Using the technique presented here one may consider more complicated cases, for example, the case of the sandwich plate with a core made of FGM viscoelastic material or the laminate plate made of viscoelastic laminae.

\subsection{Bending of a Symmetric Isotropic Plate}

Using [3] and the Laplace transform, one can reduce (2.1) and (2.2) to

$$
s \bar{D}_{\text {eff }} \Delta \Delta \bar{w}=\bar{q}_{n}-\frac{\bar{D}_{\text {eff }}}{\bar{\Gamma}} \Delta \bar{q}_{n},
$$

where $w$ is the deflection, and $q_{n}$ is the transverse load. Here we consider the symmetry of the material properties with respect to the mid-plane and $\mathbf{m}=\mathbf{0}$. Using assumption $v=$ const we transform (2.26) to the form

$$
s \bar{D}_{\text {eff }} \Delta \Delta \bar{w}=\bar{q}_{n}-\frac{2}{\lambda^{2}(1-v)} \Delta \bar{q}_{n} .
$$

Let us consider a rectangular plate $x \in[0, a], y \in[0, b]$, where $a$ and $b$ are the length and width of the plate, simple support boundary conditions, and the sinusoidal load

$$
q_{n}=Q(t) \sin \frac{\pi x}{a} \sin \frac{\pi y}{b} .
$$

Then the solution of (2.27) has the form

$$
\bar{w}=\frac{K}{\eta^{4} h^{3}} \frac{\hat{Q}}{s \bar{G}(s)} \sin \frac{\pi x}{a} \sin \frac{\pi y}{b},
$$

where

$$
\begin{aligned}
K & =1+\frac{2 \eta^{2}}{(1-v) \lambda^{2}}, \hat{Q}=\frac{\bar{Q} h^{3}}{D_{\mathrm{eff}}^{0}}, \eta=\left(\frac{\pi}{a}\right)^{2}+\left(\frac{\pi}{b}\right)^{2}, \\
D_{\mathrm{eff}}^{0} & =\frac{C_{22}+C_{33}}{G(t)} .
\end{aligned}
$$




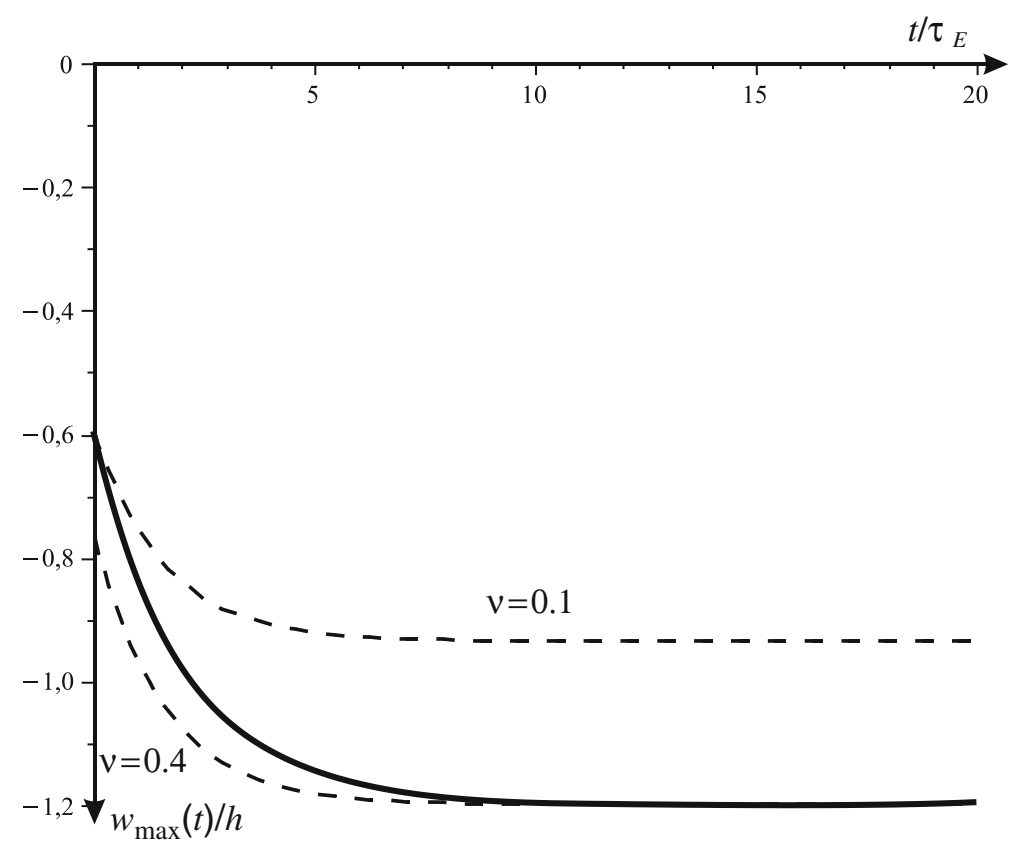

Fig. 2.3 Dimensionless maximal deflection in dependence on time: General case - solid line; Constant Poisson's ratio - dashed lines

The factor $K$ determines the maximum deflection. For the Kirchhoff's plate theory $K=1$, for the homogeneous plate modeled in the sense of Mindlin's plate theory $K=1+\frac{2 \eta}{\pi^{2}(1-v)}$. For the FGM plate the bounds for $K$ are given in [3].

For the viscoelastic plate both the qualitative and the quantitative influence of the shear stiffness is the same as in [2]. For example, let us consider an open-cell foam and following values $v=0.3, a=b, h=0.05 a, \alpha=0.9$. Using the calculation of [2] we obtain the following values of $\lambda: \lambda=0.83 h$ for $n=2, \lambda=0.82 h$ for $n=5$. The corresponding values of $K$ are given by $K \approx 1.2 \quad(n=2), K \approx$ $1.21(n=5)$. That means that for the functionally graded plates the influence of transverse shear stiffness may be significant. As well as for elastic FGM plates for the cases of other types of boundary conditions the influence of the structure of viscoelastic plate on the deflection may be greater than for the used simple support type boundary conditions. A numerical example concerning the maximal deflection vs. time is given in Fig. 2.3.

\subsection{Conclusions}

Here we presented the new model of the linear viscoelastic plates made of such FGM as polymer foam with the non-homogeneous distribution of porosity. The socalled direct approach is applied to the statement of the boundary value problem of 
the viscoelastic plates. Within this approach the plate is considered as a deformable surface. The balance laws and the constitutive equations are formulated as for $2 \mathrm{D}$ continuum. The procedure of the identification of the viscoelastic material properties is described and the example of the bending of a FGM viscoelastic plate is given. The given examples of effective relaxation functions in the cases of homogeneous, sandwich and FGM plates show that any viscoelastic plate considered as a 2D viscoelastic continuum has more complicated viscoelastic properties than the bulk material. These properties depend on the bulk properties and the through-the-thickness structure of plate, in general.

Acknowledgements The second author was supported by the RFBR with the grants No. 09-0100459, 09-01-00849 and by the DFG grant No. AL 341/33-1.

\section{References}

1. Altenbach, H., Eremeyev, V.A.: Analysis of the viscoelastic behavior of plates made of functionally graded materials. Zeitschrift für Angewandte Mathematik und Mechanik (ZAMM). 88, 332-341 (2008)

2. Altenbach, H., Eremeyev, V.A.: On the bending of viscoelastic plates made of polymer foams. Acta Mech. 204, 137-154 (2009)

3. Altenbach, H., Eremeyev, V.A.: On the time dependent behaviour of FGM plates. Key Eng. Mater. 399, 63-70 (2009)

4. Gibson, L.J., Ashby, M.F.: Cellular Solids: Structure and Properties, 2nd edn, Cambridge Solid State Science Series. Cambridge University Press, Cambridge (1997)

5. Mills, N.: Polymer Foams Handbook Engineering and Biomechanics Applications and Design. Guide Butterworth-Heinemann, Boston, MA (2007)

6. Altenbach, H.: Definition of elastic moduli for plates made from thickness-uneven anisotropic material. Mech. Solids 22, 135-141 (1987)

7. Altenbach, H.: An alternative determination of transverse shear stiffnesses for sandwich and laminated plates. Int. J. Solids Struct. 37, 3503-3520 (2000)

8. Altenbach, H.: On the determination of transverse shear stiffnesses of orthotropic plates. ZAMP. 51, 629-649 (2000)

9. Altenbach, H., Eremeyev, V.A.: Direct approach based analysis of plates composed of functionally graded materials. Arch. Appl. Mech. 78, 775-794 (2008)

10. Altenbach, H., Eremeyev, V.A.: Eigen-vibrations of plates made of functionally graded material. CMC: Comput. Mater. Continua 9, 153-178 (2009)

11. Altenbach, H.: Eine direkt formulierte lineare Theorie für viskoelasische Platten und Schalen. Ingenieur-Arch. 58, 215-228 (1988)

12. Praveen, G.N., Reddy, J.N.: Nonlinear transient thermoelastic analysis of functionally graded ceramic-metal plates. Int. J. Solids Struct. 35, 4457-4476 (1998)

13. He, X.Q., Ng, T.Y., Sivashanker, S., Liew, K.M.: Active control of FGM plates with integrated piezoelectric sensors and actuators. Int. J. Solids Struct. 38, 1641-1655 (2001)

14. Yang, J., Shen, H.S.: Dynamic response of initially stressed functionally graded rectangular thin plates. Compos. Struct. 54, 497-508 (2001)

15. Javaheri, R., Eslami, M.R.: Thermal buckling of functionally graded plates. AIAA J. 40, 162-169 (2002)

16. Arciniega, R., Reddy, J.: Large deformation analysis of functionally graded shells. Int. J. Solids Struct. 44, 2036-2052 (2007)

17. Batra, R.C.: Higher order shear and normal deformable theory for functionally graded incompressible linear elastic plates. Thin-Walled Struct. 45, 974-982 (2007)

18. Zhang, N.-H., Wang, M.-L.: Thermoviscoelastic deformations of functionally graded thin plates. Eur. J. Mech. A-Solids 26, 872-886 (2007) 
19. Lakes, R.S.: The time-dependent Poisson's ratio of viscoelastic materials can increase or decrease. Cell. Polym. 11, 466-469 (1992)

20. Lakes, R.S., Wineman, A.: On Poisson's ratio in linearly viscoelastic solids. J Elast. 85, 45-63 (2006)

21. Christensen, R.M.: Theory of Viscoelasticity An Introduction. Academic Press, New York (1971)

22. Drozdov, A.D.: Finite Elasticity and Viscoelasticity. World Scientific, Singapore (1996)

23. Tschoegl, N.W.: The Phenomenological Theory of Linear Viscoelastic Behavior. An Introduction. Springer, Berlin (1989)

24. Brinson, H.F., Brinson, C.L.: Polymer Engineering Science and Viscoelasticity. An Introduction. Springer, New York, NY (2008)

25. Riande, E., et al. (eds.): Polymer Viscoelasticity: Stress and Strain in Practice. Marcel Dekker, New York, NY (2000)

26. Timoshenko, S.P.: On the correction for shear of the differential equation for transverse vibrations of prismatic bars. Phil. Mag. Ser. 41, 744-746 (1921)

27. Reissner, E.: On the theory of bending of elastic plates. J. Math. Phys. 23, 184-194 (1944)

28. Reissner, E.: On bending of elastic plates. Q. Appl. Math. 5, 55-68 (1947)

29. Mindlin, R.D.: Influence of rotatory inertia and shear on flexural motions of isotropic elastic plates. Trans. ASME J. Appl. Mech. 18, 31-38 (1951)

30. Reissner, E.: Reflection on the theory of elastic plates. Appl. Mech. Rev. 38, 1453-1464 (1985) 